\title{
Antidepressant-Like Properties of Novel HDAC6-Selective Inhibitors with Improved Brain Bioavailability
}

\author{
Jeanine Jochems', Janette Boulden', Bridgin G Lee ${ }^{2}$, Julie A Blendy ${ }^{2}$, Matthew Jarpe ${ }^{3}$, Ralph Mazitschek ${ }^{4}$, \\ John H Van Duzer ${ }^{3}$, Simon Jones ${ }^{3}$ and Olivier Berton ${ }^{*, 1}$ \\ 'Department of Psychiatry, Perelman School of Medicine, University of Pennsylvania, Philadelphia, PA, USA; '2Department of Pharmacology, \\ Perelman School of Medicine, University of Pennsylvania, Philadelphia, PA, USA; ${ }^{3}$ Acetylon Pharmaceuticals, Boston, MA, USA; ${ }^{4}$ Center for \\ Systems Biology, Massachusetts General Hospital, Boston, MA, USA
}

\begin{abstract}
HDAC inhibitors have been reported to produce antidepressant and pro-cognitive effects in animal models, however, poor brain bioavailability or lack of isoform selectivity of current probes has limited our understanding of their mode of action. We report the characterization of novel pyrimidine hydroxyl amide small molecule inhibitors of HDAC6, brain bioavailable upon systemic administration. We show that two compounds in this family, ACY-738 and ACY-775, inhibit HDAC6 with low nanomolar potency and a selectivity of 60to I 500-fold over class I HDACs. In contrast to tubastatin A, a reference HDAC6 inhibitor with similar potency and peripheral activity, but more limited brain bioavailability, ACY-738 and ACY-775 induce dramatic increases in $\alpha$-tubulin acetylation in brain and stimulate mouse exploratory behaviors in novel, but not familiar environments. Interestingly, despite a lack of detectable effect on histone acetylation, we show that ACY-738 and ACY-775 share the antidepressant-like properties of other HDAC inhibitors, such as SAHA and MS-275, in the tail suspension test and social defeat paradigm. These effects of ACY-738 and ACY-775 are directly attributable to the inhibition of HDAC6 expressed centrally, as they are fully abrogated in mice with a neural-specific loss of function of HDAC6. Furthermore, administered in combination, a behaviorally inactive dose of ACY-738 markedly potentiates the anti-immobility activity of a subactive dose of the selective serotonin reuptake inhibitor citalopram. Our results validate new isoform-selective probes for in vivo pharmacological studies of HDAC6 in the CNS and reinforce the viability of this HDAC isoform as a potential target for antidepressant development. Neuropsychopharmacology (2014) 39, 389-400; doi:10.1038/npp.2013.207; published online 9 October 2013
\end{abstract}

Keywords: HDAC inhibitor; HDAC6; antidepressant; depression; tubulin acetylation; epigenetic

\section{INTRODUCTION}

Originally named in relation to their role in chromatin regulation, HDACs comprise a family of 11 structurallyrelated zinc-binding hydrolases, which catalyze the removal of acetyl moeties from lysine residues on proteins (Bradner et al, 2010). Deacetylation of N-terminal histone tails is the predominantly studied function of HDACs. However, recent proteomics studies have also identified a wide variety of non-histone substrates for HDACs, including transcription factors, cytoskeletal proteins, metabolic enzymes, and chaperones (Choudhary et al, 2009). These non-histone substrates are likely to contribute at least in part to the pharmacological activity of HDAC inhibitors reported in therapeutic areas like cancer, autoimmune diseases, and neuropsychiatric disorders. Early evidence implicates HDACs, in the pathophysiology and treatment of mood disorders. HDACs 2, 4, 5, 6, and 8 mRNAs levels are altered

\footnotetext{
*Correspondence: Dr O Berton, Department of Psychiatry, Perelman School of Medicine, University of Pennsylvania, 125s 31st Street, TRL22 18, Philadelphia, PA 19104-3403, USA, Tel: + I 215-573-6442, Fax: + I 215-573-2041, E-mail: bertonol@mail.med.upenn.edu Received 17 April 2013; revised I5 July 2013; accepted 2 August 20 I3; accepted article preview online 19 August 2013
}

in blood cells or post-mortem brain of patients diagnosed with mood disorders (Covington et al, 2009; Hobara et al, 2010). Sodium valproate, a drug used in the clinical treatment of mood disorders has HDAC inhibitory properties (Phiel et al, 2001). Furthermore, expression levels of various HDACs are modulated by antidepressants and mood stabilizers in neuronal and non-neuronal tissue culture systems (Chen et al, 2010; Mao et al, 2011). Finally, several HDAC inhibitors, including SAHA, sodium butyrate, and MS-275, and the acetylating agent L-acetylcarnitine have been shown to promote antidepressant-like behavioral responses in rodent models (Covington et al, 2009; Golden et al, 2013; Nasca et al, 2013; Russo and Charney, 2013). The relative contribution of histone vs non-histone HDAC substrates to these antidepressant-like effects is not known.

Recently, along with others, we identified a role for HDAC6 in models of stress adaptation and showed that HDAC6 loss of function results in an antidepressant-like phenotype (Espallergues et al, 2012; Fukada et al, 2012; Lee et al, 2012). These results point to a role of non-histone substrates in the antidepressant-like activity of pan-HDAC inhibitors. HDAC6 is a structurally and functionally unique class IIb isoform localized almost exclusively to the cytoplasm (Verdel et al, 2000) that does not appear to deacetylate histones in vivo (Zhang et al, 2003). The most abundant and 
best-characterized substrate of HDAC6 is $\alpha$-tubulin (Hubbert et al, 2002), with a number of other HDAC6 substrates identified, including Hsp90 (Kovacs et al, 2005), cortactin (Zhang et al, 2007), and beta-catenin (Li et al, 2008).

Although first-generation hydroxamates such as TSA or SAHA indiscriminately inhibit HDAC6 and class I isoforms (HDACs 1, 2, and 3; Bertrand, 2010), small molecules developed more recently such as tubacin (Haggarty et al, 2003) and tubastatin A (Butler et al, 2010) have been shown to afford more selective inhibition HDAC6. These probes have been instrumental in validating HDAC6 as a therapeutic target in models of neurodegenerative disease (d'Ydewalle et al, 2011).

Recent studies combining the use of these pharmacological probes and genetic approaches have provided indications that the physiological impact of selective HDAC6 pharmacological inhibition or loss of function in neurons diverges drastically from that of other HDACs (Rivieccio et al, 2009). In contrast to class I isoforms, the loss of function of HDAC6 does not produce toxicity, apoptosis, or major neurodevelopmental defects in rodents (Govindarajan et al, 2012; Haberland et al, 2009; Zhang et al, 2008).

Furthermore, early clinical trials with novel HDAC6 inhibitors suggest that, despite their significant biological activity, these experimental drug candidates appear to largely circumvent undesirable side-effects classically reported with broad-acting or class I-selective inhibitors (Raje et al, 2013). However, because pharmacokinetic properties of current HDAC6-selective probes are not optimized for in vivo CNS studies, it remains unclear whether systemic pharmacological inhibition of HDAC6 can produce antidepressant-like effects in animal models.

Here we report the behavioral activity of two novel small molecule inhibitors of HDAC6 with brain bioavailability, low nanomolar potency, and selectivity over class I HDACs. We provide evidence that systemic administration of these probes induces dramatic increases in brain $\alpha$-tubulin acetylation, without concurrently altering the acetylation of histones. Further, using mice with conditional loss of function of HDAC6 we show that, through their selective inhibitory activity on HDAC6 expressed in the CNS, these probes replicate the behavioral effects of acutely and chronically administered reference antidepressants.

\section{MATERIALS AND METHODS}

\section{Animals}

Male NIH Swiss mice ordered from Harlan Laboratories (Frederick, MD) at 6 weeks of age (18-20g) were group housed and acclimated for 1 week before testing. For experiments involving HDAC6 loss of function, we used a previously described flHDAC6 ${ }^{\text {nestin-cre }}$ line (Espallergues et al, 2012) with a neural cell-specific KO of HDAC6. Mice were backcrossed on C57BL/6J background for over 10 generations. Animals were allowed food ad libitum and were tested at 6-10 weeks of age during the light phase of 12-h cycle (0700-1900 hours). All protocols were approved by the University of Pennsylvania Institutional Animal Care and Use Committee.

\section{Drugs}

ACY-738 ( $N$-hydroxy-2-(1-phenylcycloproylamino)pyrimidine-5-carboxamide) and ACY-775 (2-((1-(3-fluorophenyl)- cyclohexyl)amino)- $N$-hydroxypyrimidine-5-carboxamide) were synthesized by ChemPartner (Shanghai, China) and obtained from Acetylon Pharmaceuticals. Other drugs used: tubastatin A, a gift from A Kozikowski (University of Illinois, Chicago); trichostatin A (TSA, Sigma-Aldrich, St Louis, MO); fluoxetine- $\mathrm{HCl}$ (AK Scientific, Union City, CA); citalopram- $\mathrm{HBr}$ (AK Scientific); chlordiazepoxide- $\mathrm{HCl}$ (CDP, Sigma-Aldrich). For in vitro assays, drugs were dissolved and diluted in assay buffer (50 mM HEPES, $\mathrm{pH}$ 7.4, $100 \mathrm{~mm} \mathrm{KCl}, 0.001 \%$ Tween-20, 0.05\% BSA, and $20 \mu \mathrm{m}$ tris) to sixfold the final concentration. For pharmacokinetic analyses, drugs were dissolved in 10\% DMAC, 10\% solutol $\mathrm{HS} 15$, and $80 \%$ saline at a dose of $5 \mathrm{mg} / \mathrm{kg}$. For in vivo behavioral and biochemical assays, all drugs were dissolved in DMSO and diluted to a final concentration of $0.75 \%$ in $0.9 \%$ saline. Owing to limited solubility, the $50 \mathrm{mg} / \mathrm{kg}$ dose of ACY-775 was in a suspension state when injected. All intraperitoneal (i.p.) injections were administered at a volume of $10 \mathrm{ml} / \mathrm{kg}$.

\section{HDAC Enzymatic Assays}

Potency of each compound was determined using recombinant enzymes and a kinetic method described previously (Bradner et al, 2010). Briefly, N terminal GST-tagged HDAC enzymes (BPS Biosciences, San Diego, CA) expressed in a baculoviral system and purified by affinity chromatography and gel filtration were diluted to 1.5 -fold final concentration in assay buffer and pre-incubated with test compounds for $10 \mathrm{~min}$ before the addition of substrate, FTS (HDAC1, HDAC2, HDAC3, and HDAC6) or MAZ-1675 (HDAC4, HDAC5, HDAC7, HDAC8, and HDAC9). For studies with HDAC6 N- and C-terminal catalytic mutants, point-mutations H216A and H611A were used, respectively (Haggarty et al, 2003). The amount of substrate for each enzyme was equal to the Michaelis constant $\left(K_{\mathrm{m}}\right)$, as determined by a titration curve. The enzymatic reaction was monitored over $30 \mathrm{~min}$ for release of 7-amino-4-methoxy-coumarin after deacetylation of the lysine side chain in the peptide substrate and the linear rate of the reaction was calculated.

\section{Pharmacokinetic Profiling}

For PK analyses tubastatin A, ACY-738 and ACY-775 were dosed cassettely and measured in plasma and brain by liquid chromatography/tandem mass spectrometry. Plasma and brain were collected from mice killed 5, 15, 30, and $60 \mathrm{~min}$ after treatment. Drugs were extracted from brain and plasma by protein precipitation using 50:50 acetonitrile:methanol and analyzed using a HPLC/MS/MS method using electrospray ionization in positive mode as previously reported (Santo et al, 2012). The lower limit of quantification for all compounds was $3 \mathrm{ng} / \mathrm{ml}$. PK parameters (concentrations, $\mathrm{T}_{1 / 2}, \mathrm{AUC}_{\text {Brain }} / \mathrm{AUC}_{\text {Plasma }}$ ) were estimated by non-compartmental model using WinNonlin (Pharsight, Sunnyvale, CA).

\section{Cell Culture}

Undifferentiated RN46A-B14 cells, a line of immortalized rat raphe neuronal precursors, were grown as previously described (White et al, 1994). They were treated with $2.5 \mu \mathrm{M}$ ACY-738, ACY-775, tubastatin A, or $0.6 \mu \mathrm{m}$ TSA, or vehicle 
(0.1\% DMSO) for $4 \mathrm{~h}$. Samples were processed using Epigentek (Farmingdale, NY) histone extraction kit and quantified using protein assay (Bio-Rad, Hercules, CA).

\section{Tissue Harvest}

Brains were harvested after cervical dislocation and decapitation, flash frozen, and stored at $-80{ }^{\circ} \mathrm{C}$ until use. Needle punches from cortex (bregma $2.22 \mathrm{~mm}$ ), hippocampus (bregma $-1.34 \mathrm{~mm}$ ), dorsal raphe nucleus (DRN; bregma -4.36), and cerebellum (bregma - 5.34) were collected. Extracts from whole brains and punches were harvested and quantified as above.

\section{Immunoblotting}

Standard immunoblotting procedures were used. Antibodies: total $\alpha$-tubulin $(1: 2000$, Rockland Immunochemicals, Gilbertsville, PA), acetyl $\alpha$-tubulin (1:20 000, Sigma-Aldrich), acetyl lysine 9 histone H3 (1:500, Abcam, Cambridge, MA), and total H3 (1:1000, Abcam). Blots were imaged using LiCOR (Lincoln, NE) Odyssey system and quantified using Image $(\mathrm{NIH}$, Bethesda, MD).

\section{Chromatin Immunoprecipitation (ChIP)}

Mice were subchronically treated with ACY-738 (5 mg/kg), sodium butyrate $(1.2 \mathrm{~g} / \mathrm{kg})$, or vehicle. Chromatin extraction and ChIP was performed as previously reported (Zhang et al, 2005). Hippocampus was harvested $30 \mathrm{~min}$ after last injection and immediately crosslinked in $1.1 \%$ formaldehyde/PBS for $10 \mathrm{~min}$. Immunoprecipitation with IgG or anti-AcH3K9 (Abcam) antibodies on $5 \mu \mathrm{g}$ of chromatin was performed as reported previously (Kennedy et al, 2013). QPCR was completed on precipitated chromatin for the promoter sequences of interest (BDNF IV and cFOS; Guan et al, 2009). Promoter enrichment was calculated using primers flanking 18S rRNA loci as a control for both genomic, input DNA, and precipitated DNA. Primer sequences are available on request.

\section{Open-Field Activity}

Mice were injected with ACY-738 or ACY-775 at 5, 10, or $50 \mathrm{mg} / \mathrm{kg}$ or vehicle and allowed to explore. Activity was recorded using the Photobeam Activity System (PAS, San Diego Instruments, San Diego, CA).

\section{Home Cage Activity}

Activity was recorded using an infrared beam, home cage activity system from Med Associates (St Albans, VT) immediately following i.p. injection of $50 \mathrm{mg} / \mathrm{kg}$ ACY -738 or vehicle.

\section{Marble-Burying}

Fifteen minutes after injection, mice were placed in a standard mouse cage with $6 \mathrm{~cm}$ bedding and $20(4 \times 5)$ clear glass marbles. After $15 \mathrm{~min}$, an investigator blinded to the treatment counted the number of marbles buried to at least three-fourths.
Elevated Plus Maze (EPM)

Fifteen minutes after injection, mice were tracked in the EPM (Lister, 1987) using TopScan software (Clever Sys, Reston, VA).

\section{Novelty-Induced Hypophagia (NIH)}

$\mathrm{NIH}$ was performed as previously described (Merali et al, 2003) using peanut butter chips as a palatable food. Mice were tested $30 \mathrm{~min}$ after injection for latency to eat in a novel environment including an unfamiliar scent and bright (600 lux) lighting.

\section{Tail Suspension Test (TST)}

Mice were tested for immobility in the TST using a rig from Med-Associates. At $30 \mathrm{~min}$ or $2 \mathrm{~h}$ after i.p. injection of ACY$738(5,50 \mathrm{mg} / \mathrm{kg}), \mathrm{ACY}-775(5,50 \mathrm{mg} / \mathrm{kg})$, and citalopram $(0.5,2,20 \mathrm{mg} / \mathrm{kg})$, a combination of the previous, or vehicle, mice were attached to the test rig and time immobile over $6 \mathrm{~min}$ was recorded.

\section{Chronic Social Defeat (CSD) and Social Interaction Testing}

Social defeat was conducted as previously described (Berton et al, 2006; Golden et al, 2011). For stratification of resilient/ vulnerable sub-population after social defeat exposure, we used the score of the lowest-interacting undefeated control mouse as a cutoff. Any defeat-exposed mouse that spent over $51.8 \mathrm{~s}$ in the interaction zone with target present was categorized as resilient.

\section{Statistics}

Open-field data were analyzed using a two-way ANOVA with repeated factor, followed by Bonferroni post-tests. For other data sets, one-way ANOVA followed by NeumannKeuls post-hoc test or $t$-test were done as appropriate. Statistical analyses were conducted using GraphPad Prism 5.0a (Graph Pad Software, San Diego, CA). Drug effects on the proportion of resilient mice after social defeat were computed using $z$-test in Excel (Microsoft, Redmond, WA).

\section{RESULTS}

ACY-738 and ACY-775 demonstrate inhibitory activity against recombinant HDAC6 with IC50 values of 1.7 and $7.5 \mathrm{nM}$, respectively, with respective average selectivity over class I HDACs being 100- and 700-fold (Table 1, Figures $1 \mathrm{~b}-\mathrm{d}$ ). For comparison, the reference HDAC6 inhibitor tubastatin A showed an $\mathrm{IC}_{50}$ for HDAC6 of $18 \mathrm{~nm}$ and displayed an average 200-fold selectivity over class I HDACs (Figure 1a). ACY-775 had minimal activity against all other class II HDAC isoforms $\left(\mathrm{IC}_{50}>1 \mu \mathrm{M}\right)$. Activity of ACY-738 and ACY-775 in in vitro assays was fully explained by inhibition of the $\mathrm{C}$ terminal catalytic domain of HDAC6, as deacetylase activity of a point-mutant with inactive $\mathrm{N}$-terminal domain $(\mathrm{H} 216 \mathrm{~A})$ but intact C-terminal domain was comparable to HDAC6 WT and fully inhibited by ACY738. In contrast, the HDAC6 C-terminal catalytic mutant 
was almost completely devoid of deacetylase activity (Supplementary Figure S1).

In vivo biodistribution profiles of ACY-738, ACY-775, and tubastatin A were examined after acute dosing at 5 or $50 \mathrm{mg} / \mathrm{kg}$ over $2 \mathrm{~h}$. At $t=30 \mathrm{~min}$ after acute $50 \mathrm{mg} / \mathrm{kg}$ injection, respective plasma levels of ACY-738 and ACY775 were $515 \mathrm{ng} / \mathrm{ml}(1.9 \mu \mathrm{M})$ and $1359 \mathrm{ng} / \mathrm{ml}(4.1 \mu \mathrm{M})$. Elimination from plasma was rapid, with plasmatic halflife of $12 \mathrm{~min}$ and concentration below $10 \mathrm{ng} / \mathrm{ml}$ after $2 \mathrm{~h}$. Nevertheless, areas under concentration time curves for brain and plasma $\left(\mathrm{AUC}_{\mathrm{Brain}} / \mathrm{AUC}_{\text {Plasma }}\right)$ calculated over $2 \mathrm{~h}$ for both ACY-738 and ACY-775 led to ratios >1 (Table 1). In comparison, tubastatin A exhibited a longer plasmatic half-life of $2 \mathrm{~h}$, but a more limited brain penetration, with $\mathrm{AUC}_{\mathrm{Brain}} / \mathrm{AUC}_{\text {Plasma }}$ ratio of 0.18 . Taken together, these results suggest that despite their short half-life, ACY-738 and ACY-775 rapidly distribute to the brain leading to a total drug exposure in CNS comparable to that of peripheral tissues.

In contrast to the class I isoforms HDAC2 and 3, which are expressed highly and ubiquitously in the mouse brain, HDAC6 mRNA expression appears restricted to a small number of brain areas, with highest signal observed in DRN (Figure 1f). In RN46A-B14 cells treated with TSA $(0.6 \mu \mathrm{M})$, tubastatin A $(2.5 \mu \mathrm{M})$, ACY-738 $(2.5 \mu \mathrm{M})$, or ACY-775 $(2.5 \mu \mathrm{M})$, western blot analyses at $4 \mathrm{~h}$ after treatment revealed increases in the acetylated (lysine 40) fraction of $\alpha$-tubulin $\left(\mathrm{F}_{4,9}=48.69, P<0.0001\right)$. No changes in total $\alpha$-tubulin expression were detected (Figure 2a). Although TSA, led to a $94 \%$ increase in acetylation of histone $\mathrm{H} 3$ at lysine $9\left(\mathrm{~F}_{4,5}=22.21, P=0.0022\right)$, no significant change in histone $\mathrm{H} 3 \mathrm{~K} 9$ acetylation was observed following treatment with ACY-738, ACY-775, or tubastatin A (Figure 2a).

Although tubastatin A resulted in an increase in $\alpha$-tubulin acetylation of about $400 \%$ in tissue culture (Figure $2 \mathrm{a}$ ), and produced $268 \%$ increased $\alpha$-tubulin acetylation in heart $(P<0.05)$ upon single systemic administration in vivo $(10 \mathrm{mg} / \mathrm{kg}, 2.7 \mathrm{~mm})$, it did not significantly change levels of $\alpha$-tubulin acetylation in whole-brain lysates (Figure $2 b$ ). In contrast, ACY $-738(5 \mathrm{mg} / \mathrm{kg}, 1.9 \mathrm{~mm})$ and ACY -775 $(50 \mathrm{mg} / \mathrm{kg}, 15 \mathrm{~mm})$, both led to significant increase in $\alpha$-tubulin acetylation in whole-brain lysates. Changes were significant at $30 \mathrm{~min}\left(\mathrm{~F}_{3,13}=163.4, P<0.0001\right), 1 \mathrm{~h}$ $\left(\mathrm{F}_{3,13}=163.9, \quad P<0.0001\right), \quad$ and $4 \mathrm{~h} \quad\left(\mathrm{~F}_{3,14}=4.703\right.$, $P=0.0179)$ after administration.

When ACY-738 (5 mg/kg) or ACY-775 (50 mg/kg) were administered repeatedly in wild-type mice at $24 \mathrm{~h}, 4 \mathrm{~h}$, and $30 \mathrm{~min}$ before killing, significant increases in $\alpha$-tubulin acetylation were observed in all tested brain regions (Figure 2c): cortex, $\mathrm{F}_{2,7}=582.5, P<0.0001$; hippocampus, $\mathrm{F}_{2,7}=260.4, P<0.0001 ; \mathrm{DRN}, \mathrm{F}_{2,7}=54.00, P<0.0001 ;$ and cerebellum, $\mathrm{F}_{2,7}=136.2, P<0.0001$. In contrast, an identical treatment regimen in $\mathrm{KO}$ mice did not produce increases in $\alpha$-tubulin acetylation over baseline levels (Figure 2c). Of note, as reported previously (Espallergues et al, 2012), these mutant mice have markedly enhanced baseline acetylated $\alpha$ tubulin $(1.98 \pm 0.2$ acetyl/total $)$ when compared with their WT littermates $(0.5 \pm 0.06 ; P<0.0001)$.

As reported above in cell culture, treatment with ACY-738 and ACY-775 in vivo did not significantly alter acetylation of histone 3 at lysine 9 in western blot. In addition, ChIP revealed no concurrent changes in histone $\mathrm{H} 3 \mathrm{~K} 9$ acetylation 


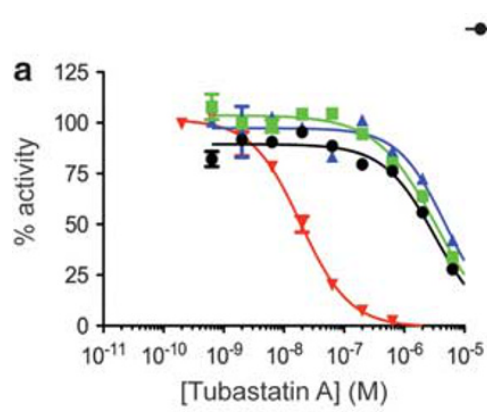

- HDAC1 - HDAC2 \& HDAC3 - HDAC6
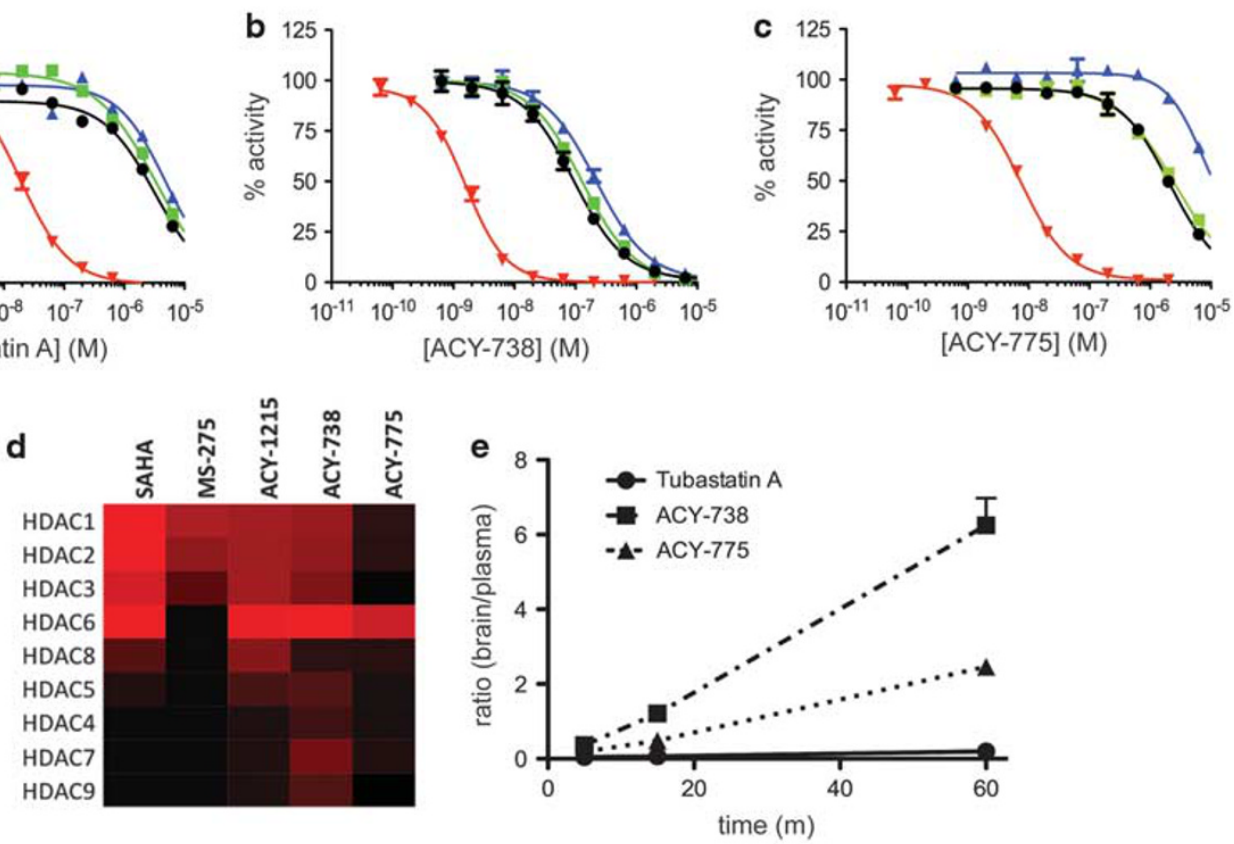

f
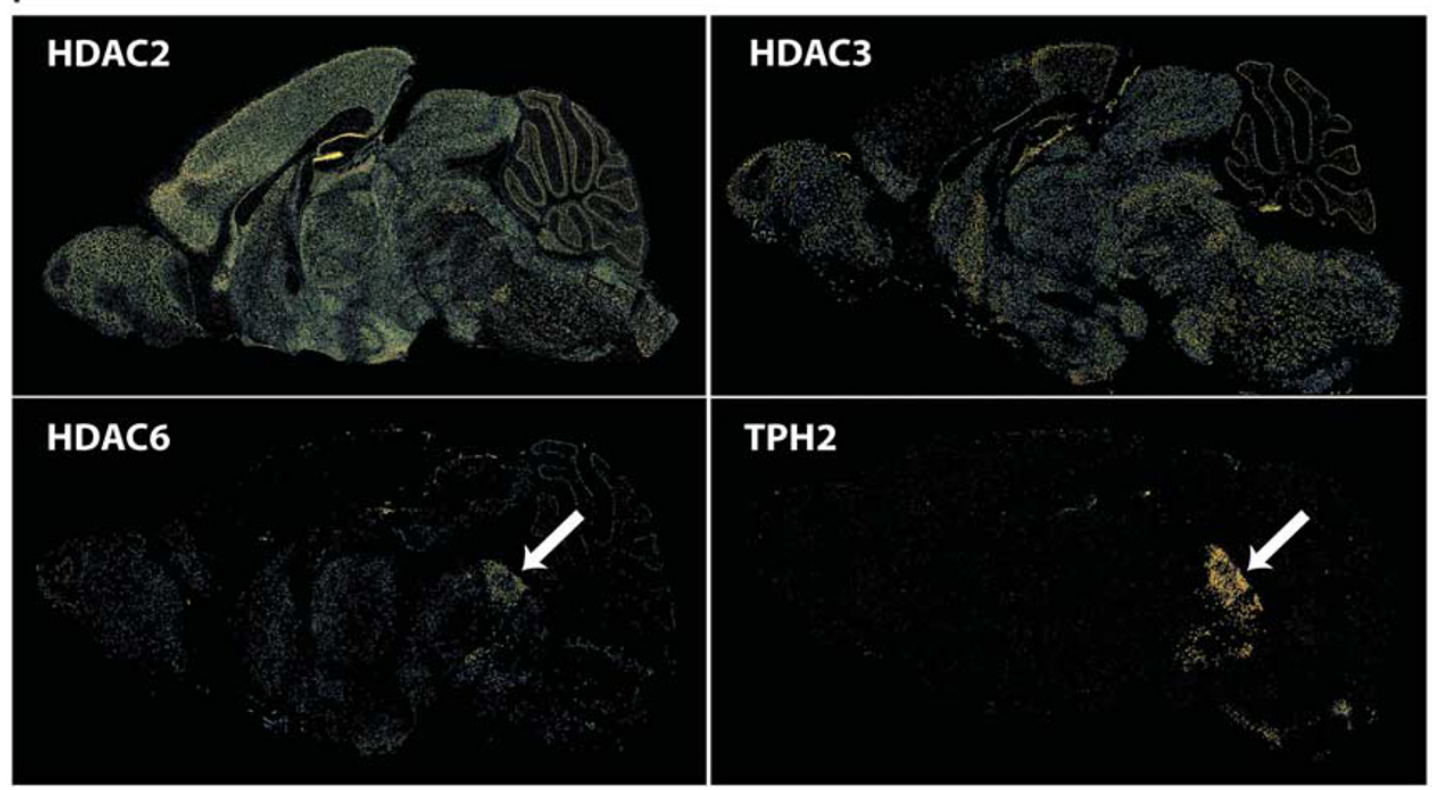

Figure I Selectivity, potency, and pharmacokinetic properties of HDAC6 inhibitors used in this study. Top row (a-c): dose-dependent enzymatic inhibition of recombinant HDACI, HDAC2, HDAC3, and HDAC6 incubated with (a) tubastatin A, (b) ACY-738, or (c) ACY-775. Middle row: (d) heat map summary of IC50 values for the novel inhibitors ACY-738 and ACY-775 and reference compounds SAHA, MS-275, and ACY-I2I5 ( $n=2$ per condition). Brighter reds denote higher inhibitory potency of compounds on corresponding HDAC isoform, black background indicates lack of activity. (e) Ratios of brain concentration to plasma concentration over time after acute $5 \mathrm{mg} / \mathrm{kg}$ i.p. injection. Bottom rows (f): distribution of HDAC2, HDAC3, HDAC6, and TPH2 mRNA as seen after in situ hybridization on sagittal views of the mouse brain. Average expression intensities range from low (blue) to high (red). Pictures courtesy of the Allen Mouse Brain Atlas (Allen Institute for Brain Science; http://www.brain-map.org/).

enrichment at promoter DNA within the hippocampus following repeated i.p. treatment with $5 \mathrm{mg} / \mathrm{kg}$ ACY-738. This is contrast to treatment with a behaviorally active dose of sodium butyrate $(1.2 \mathrm{~g} / \mathrm{kg})$, a class I histone deacetylase inhibitor, which led to increases in enrichment of acetylated $\mathrm{H} 3 \mathrm{~K} 9$ at the promoter of activity-induced neuronal genes, namely cFOS and BDNF promoter 4 (Figure $2 \mathrm{~d}$ and e).

Common antidepressants have been shown to increase ambulation in a novel environment (Brocco et al, 2002). We tested the effects of acute administration of ACY-738, ACY775 , and tubastatin A on exploration in a novel open-field arena. When injected after $1 \mathrm{~h}$ of habituation, tubastatin A did not change exploratory activity at $10 \mathrm{mg} / \mathrm{kg}$. However, we observed rapid, dose-dependent hyperlocomotor effects after treatment with $50 \mathrm{mg} / \mathrm{kg}$ ACY-738 $\left(\mathrm{F}_{4,132}=84.63\right.$, $P<0.0001$; Figures $3 \mathrm{a}$ and $\mathrm{c})$ or ACY775 $\left(\mathrm{F}_{4,174}=7.265\right.$, $P<0.0001$; Figure $3 \mathrm{~b}$ and $\mathrm{c})$. There was a significant effect of time (ACY-738: $\mathrm{F}_{5,132}=84.63, \quad P<0.0001 ;$ ACY-775: 
$\left.\mathrm{F}_{5,174}=28.71, P<0.001\right)$, with effect peaking in the first hour after injection and returning to baseline by $2 \mathrm{~h}$, consistent with brain biodistribution profiles. The same doses of ACY-775 or ACY-738 did not significantly alter open-field exploration in mice with neural cell-selective $\mathrm{KO}$ HDAC6 (Figures 3a-c). Distance in the center of the open field trended toward an increase, with ACY-738 (602 \pm 96 beam breaks) and ACY-775 (419 \pm 73 beam breaks) treated mice traveling a greater distance in the center than those treated with vehicle ( $177 \pm 71$ beam breaks). Furthermore, a $50 \mathrm{mg} / \mathrm{kg}$ dose of ACY-738 failed to produce an enhancement of locomotor activity in WT mice tested in a home

a

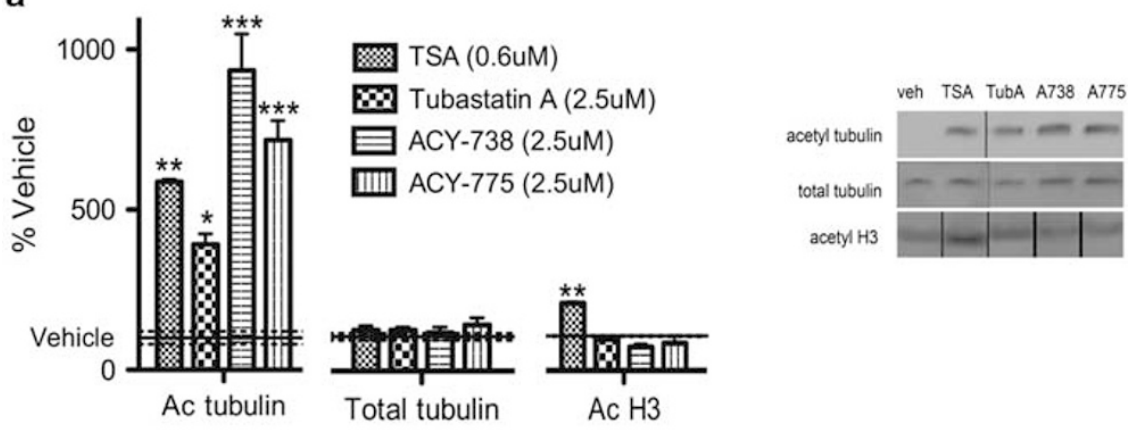

b
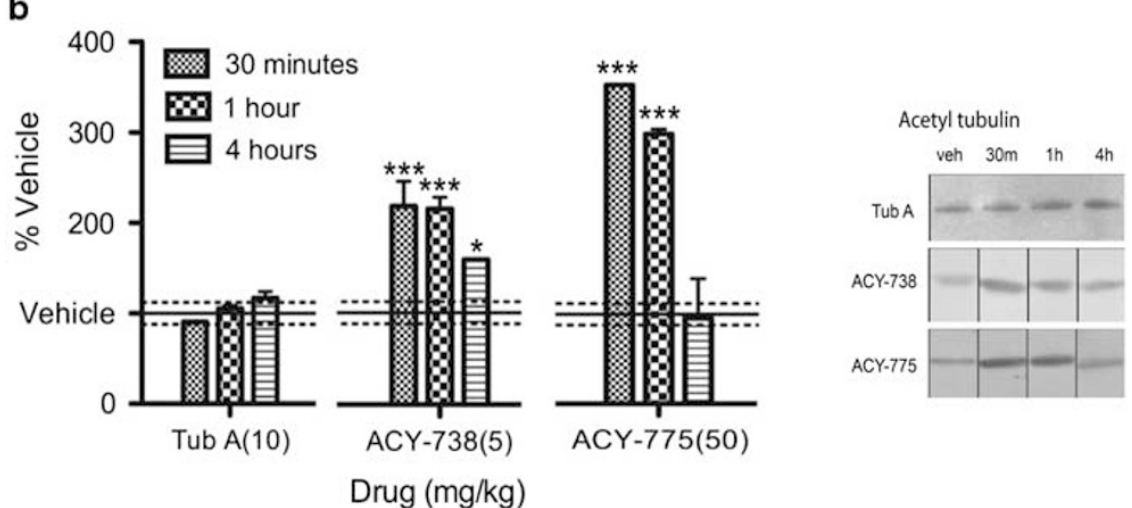

c

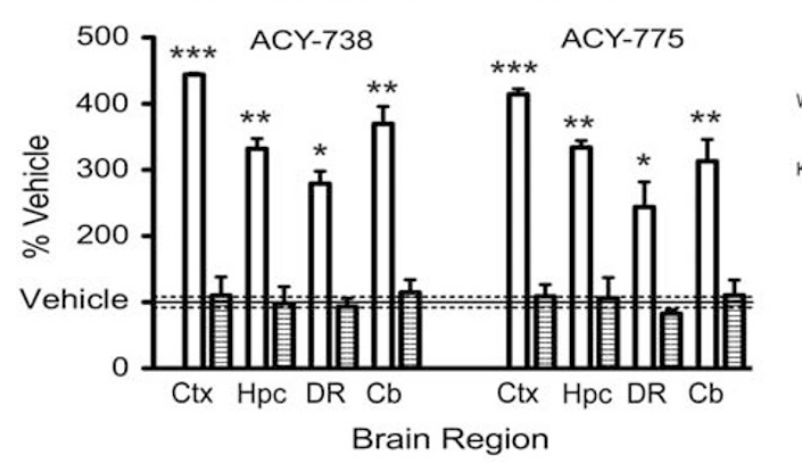

Acetyl tubulin

d

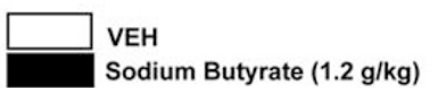

BDNF P4 Promoter

cFOS Promoter
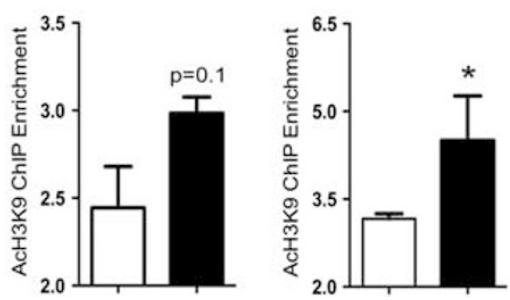

e

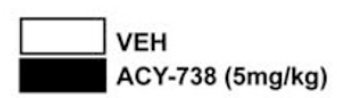

BDNF P4 Promoter

cFOS Promoter
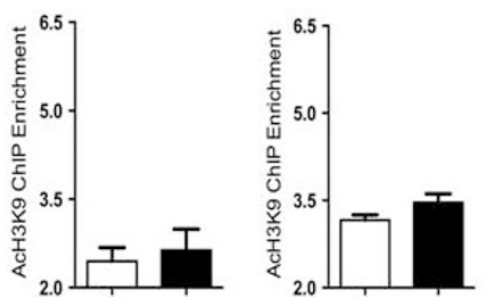
cage environment (veh $=4273 \pm 425$ beam breaks; ACY$738=3468 \pm 711$ beam breaks; $F_{1,232}=0.9447, P=0.36$ ), suggesting that the effect reflects a disinhibition of exploratory behavior under neophobic condition, rather than a nonspecific elevation of motor function.

In the marble-burying task, a test sensitive to both anxiolytics (Broekkamp et al, 1986) and antidepressants (Albelda and Joel, 2012), treatment with ACY-738 and ACY-775 significantly reduced the number of marbles buried, to a similar degree as a non-sedating $(10 \mathrm{mg} / \mathrm{kg})$ dose of the common anxiolytic CDP (Figure 3d; $\mathrm{F}_{3,50}=$ $3.699, P=0.0176)$. In addition, ACY-738 given acutely at a dose of $50 \mathrm{mg} / \mathrm{kg}$ significantly decreased latency to eat in the NIH test $(P<0.05)$, although this effect was not as potent as that of $\operatorname{CDP}(P<0.01$; Figure $3 \mathrm{e})$. In the EPM, a test sensitive to anxiolytics but not antidepressants, neither ACY-738 nor ACY-775 produced behavioral changes, whereas CDP markedly increased the time in the open arms of the maze (Figure 3f; $\mathrm{F}_{3,16}=7.498$, $P=0.0024)$.

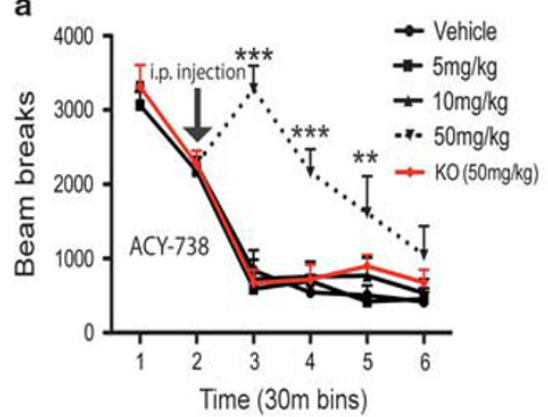

d

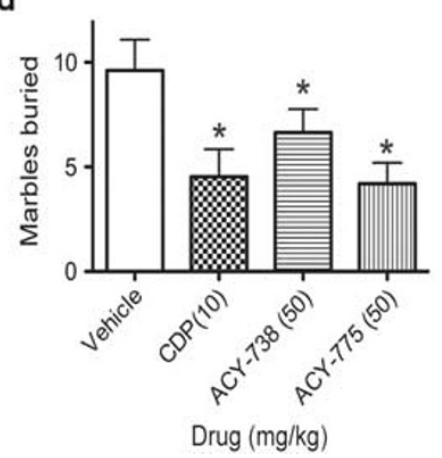

b

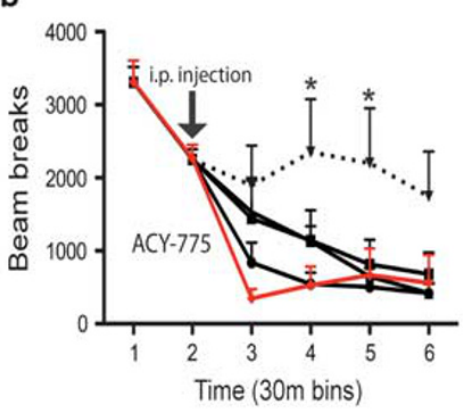

e

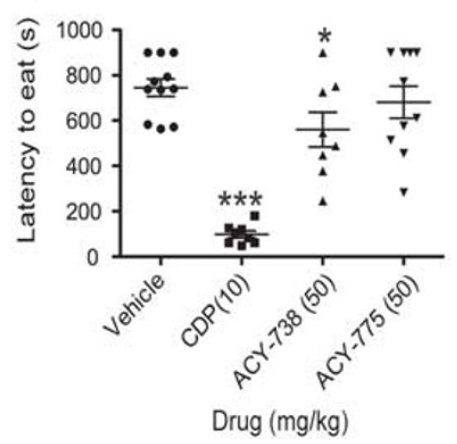

C

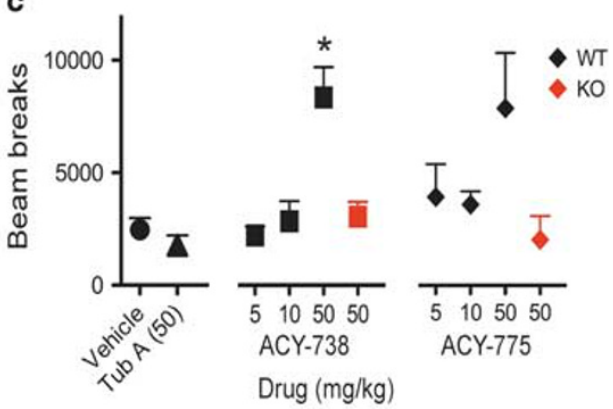

f

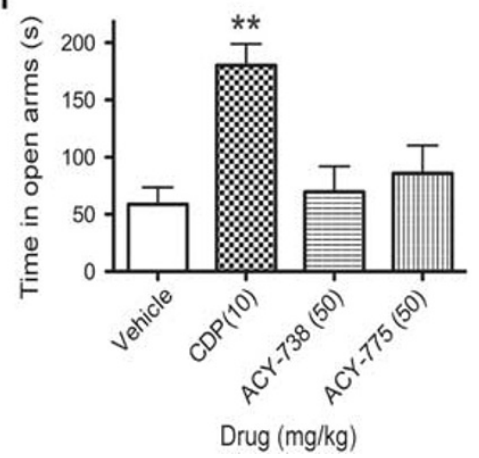

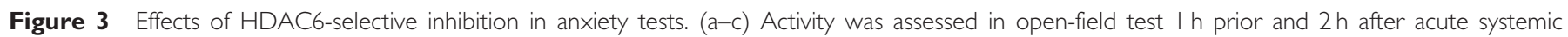

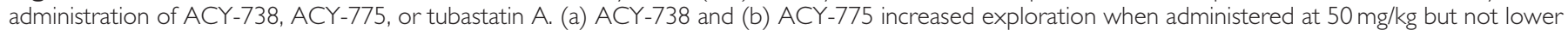

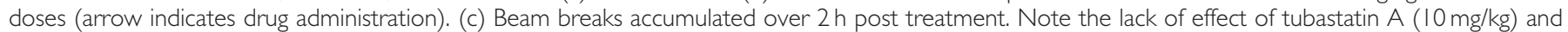

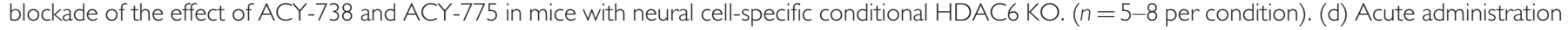

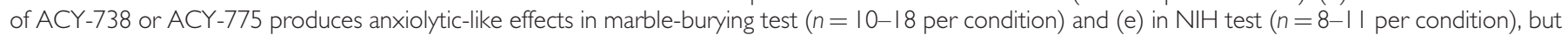
(f) has no effect in elevated plus maze ( $n=5$ per condition). Data expressed as mean values \pm SEM. * $P<0.05$, ** $P<0.0$ I, **** $<0.00$ I vs vehicle.

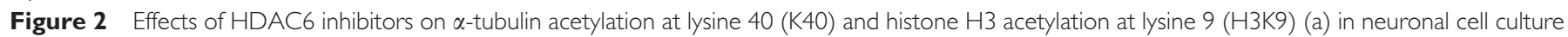

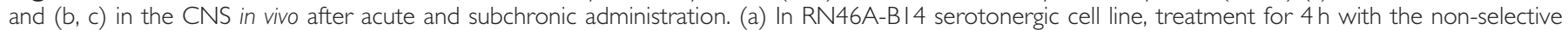

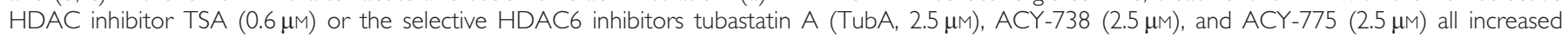

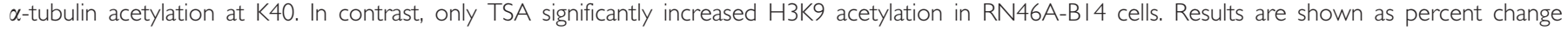

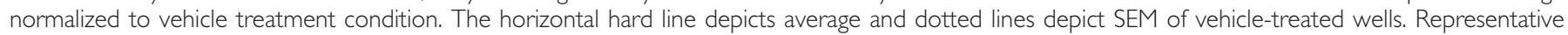

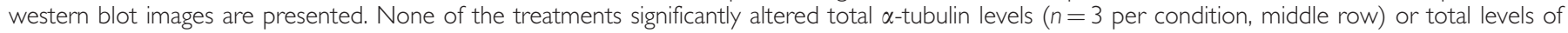

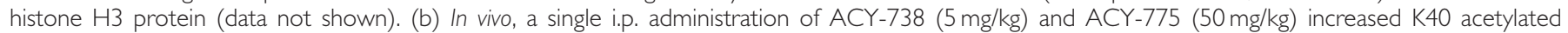

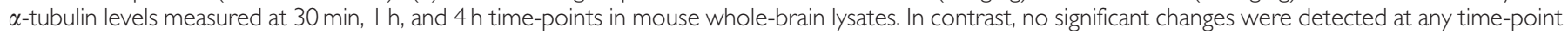

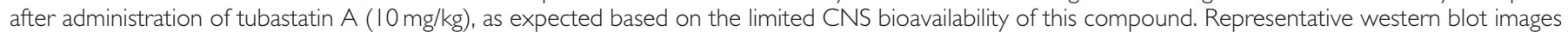

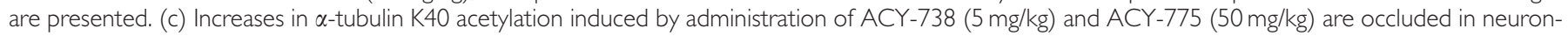

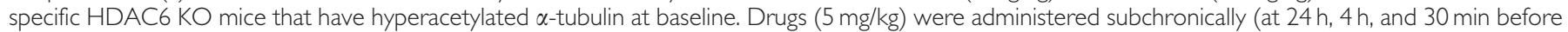

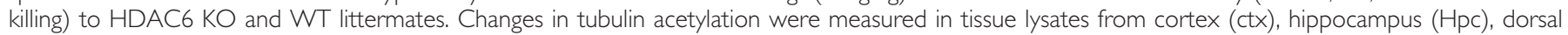

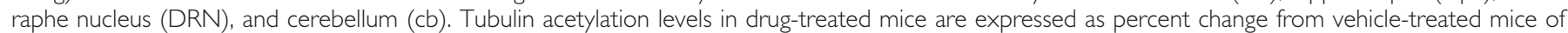

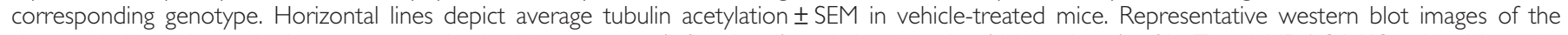

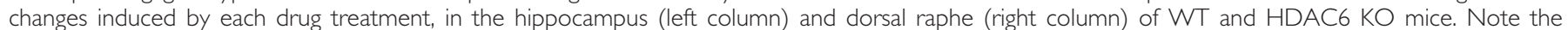

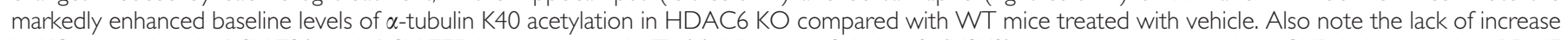

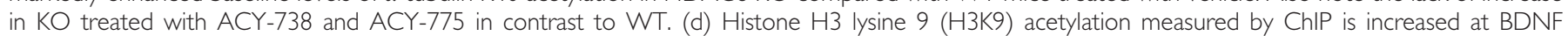

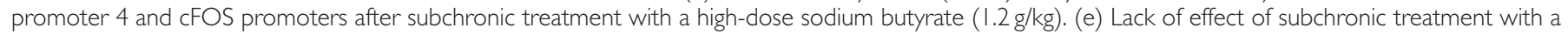

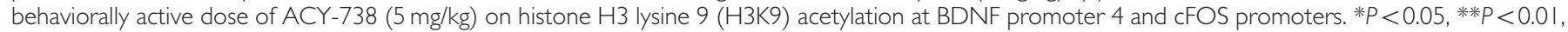
***** $P<0.001$ vs vehicle $(n=2-3$ per condition). 
a

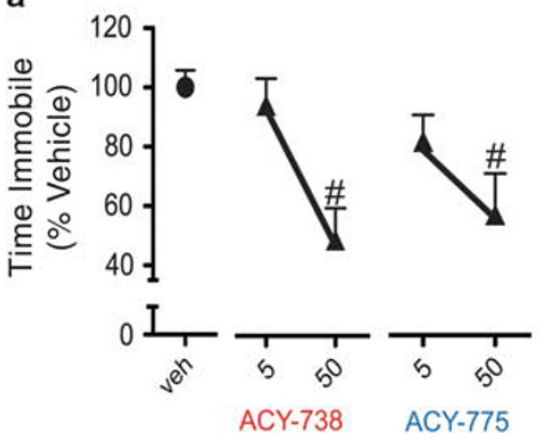

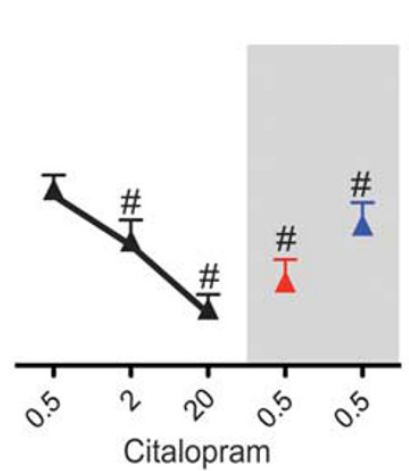

Citalopram

Treatment $(\mathrm{mg} / \mathrm{kg})$

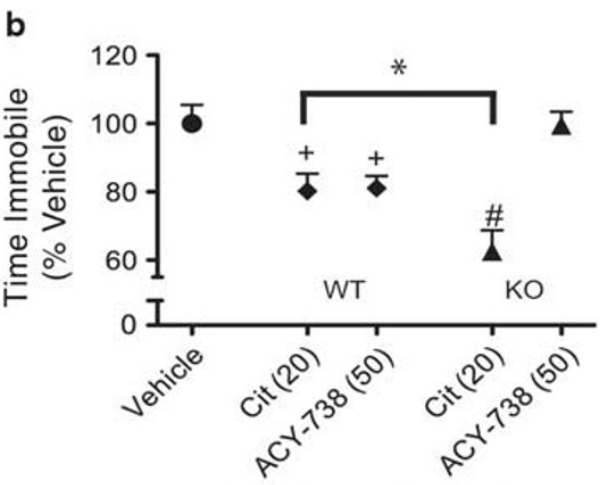

Treatment $(\mathrm{mg} / \mathrm{kg})$

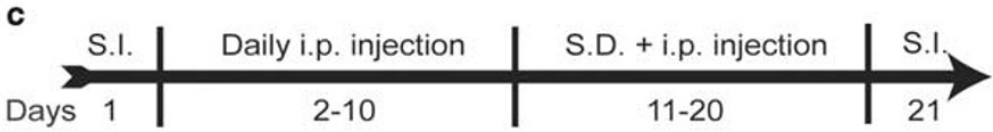

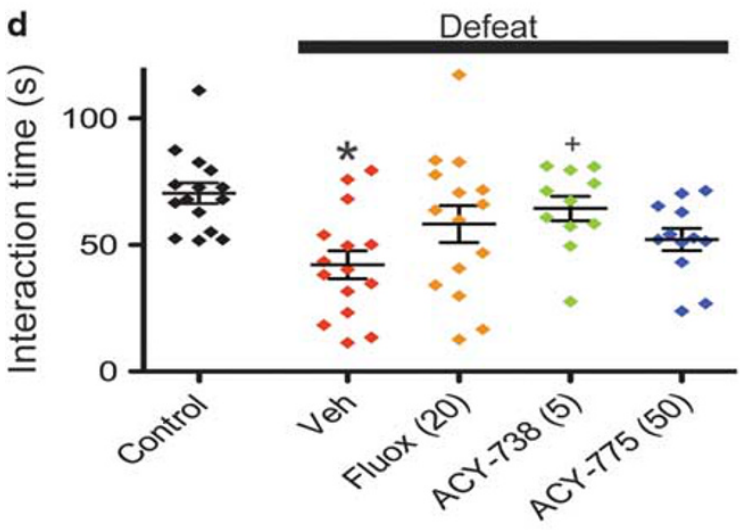

Treatment $(\mathrm{mg} / \mathrm{kg})$

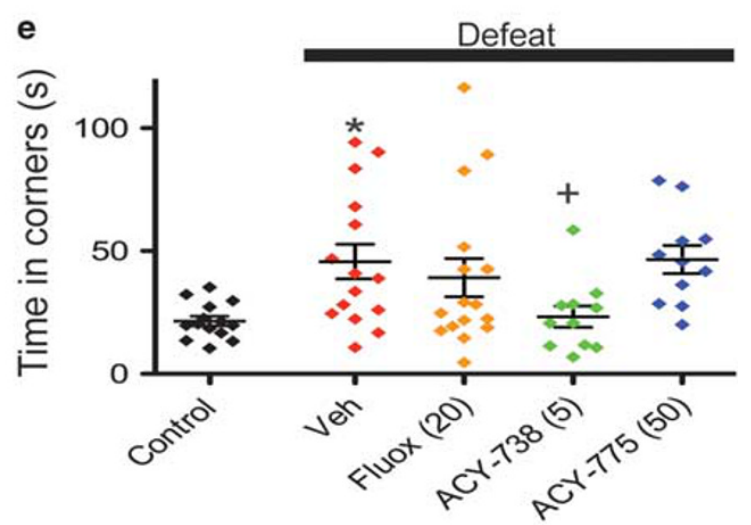

Treatment $(\mathrm{mg} / \mathrm{kg})$

$f$
\begin{tabular}{|c|c|}
\hline Treatment & $\%$ Resilient \\
\hline Vehicle & 27 \\
\hline Fluoxetine & $60^{\#}$ \\
\hline ACY-738 & $81^{\#}$ \\
\hline ACY-775 & 58 \\
\hline
\end{tabular}

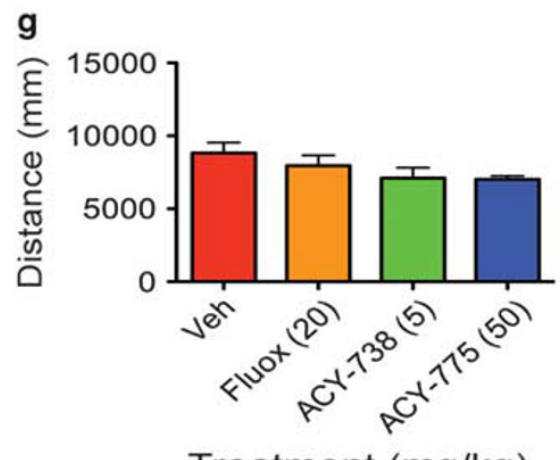

Treatment $(\mathrm{mg} / \mathrm{kg})$

Figure 4 HDAC6 inhibitors ACY-738 and ACY-775 have antidepressant-like properties. (a) In the TST acute administration of ACY-738 and ACY-775 produce anti-immobility effects in NIH Swiss mice resembling those of the reference antidepressant citalopram. Effects are seen $30 \mathrm{~min}$ after acute $50 \mathrm{mg} / \mathrm{kg}$ i.p. injection, but not for lower dose $(5 \mathrm{mg} / \mathrm{kg})$. Combination of subactive doses of HDAC6-selective inhibitors $(5 \mathrm{mg} / \mathrm{kg})$ and citalopram $(0.5 \mathrm{mg} / \mathrm{kg})$ produces robust potentiation of anti-immobility activity ( $n=8-29$ per condition). (b) In C57BL/6) mice, the anti-immobility effect of ACY-738 is blocked by a neural cell-selective KO of HDAC6, whereas citalopram effect is potentiated by HDAC6 KO. No difference was observed between HDAC6 WT and KO when treated with vehicle ( $n=6-15$ per condition). (c) Timeline showing treatment period, social defeat and social interaction (SI) testing. Chronic treatment with HDAC6-selective inhibitors prevents development of avoidance after social defeat as observed in (d) an increased average interaction time and (e) a decrease in time spent in the corners of the test arena, as compared with vehicle-treated mice that underwent social defeat. ( $f$ ) In addition, treatment with SSRI or HDAC6 inhibitors results in a greater percentage of resilient mice compared with vehicle treatment. (g) No change in total distance traveled during the test period was observed ( $n=1|-| 5$ per condition). Data expressed as mean values \pm SEM. ${ }^{+} P<0.05$ vs vehicle, ${ }^{\#} P<0.01$ vs vehicle, $* P<0.01$ vs undefeated control.

In the TST (Figure 4a), we observed a significant effect of drug/dose $\left(F_{12,140}=9.368, P<0.0001\right)$. Significant reductions in immobility were detected at $50 \mathrm{mg} / \mathrm{kg}$ with either
ACY-738 $(P<0.001)$ or ACY-775 $(P<0.01)$. No significant effects of either drug were seen after a $5 \mathrm{mg} / \mathrm{kg}$ dose. Administration of the SSRI citalopram led to significant 
decreases in time immobile at $2 \mathrm{mg} / \mathrm{kg}(P<0.001)$ and $20 \mathrm{mg} / \mathrm{kg}(P<0.001)$, but not at $0.5 \mathrm{mg} / \mathrm{kg}$. At $2 \mathrm{~h}$ after injection, treatment with $20 \mathrm{mg} / \mathrm{kg}$ citalopram $(P<0.001)$ or $50 \mathrm{mg} / \mathrm{kg}$ ACY-738 $(P<0.01)$ still had significant activity.

To test whether anti-immobility effect of citalopram and ACY-738 are dependent on intact HDAC6 protein levels, we administered citalopram to mice with neural KO of HDAC6 or their WT littermates bred on a C57BL/6J background (Figure 4b). In line with previous reports comparing C57BL/6J with NIH Swiss mice in TST (Lucki et al, 2001), we found that, while anti-immobility effects were still significant, C57BL/6J WT had higher baseline immobility $(P<0.0001)$ and were less responsive to both citalopram $(P<0.0001)$ and ACY-738 $(P<0.01)$ than NIH Swiss mice. In contrast, the anti-immobility effect of citalopram was significantly greater in HDAC6 KO mice than WT littermates $\left(\mathrm{F}_{4,47}=7.902, P<0.0001\right)$, in line with a previous report showing that the activity of another SSRI, fluoxetine, is amplified in HDAC6 KOs (Fukada et al, 2012). However, when anti-immobility activity of ACY-738 was tested in HDAC6 KO mice, no significant effect was observed, indicating that intact HDAC6 protein is required for ACY738 action in TST.

To further examine the interactions between SSRI and HDAC6, we tested the effects of a combined administration of citalopram and HDAC6 inhibitors in NIH Swiss mice (Figure 4a). We found significant anti-immobility effects of a subactive dose of citalopram $(0.5 \mathrm{mg} / \mathrm{kg})$ when administered in combination with ACY-738 or ACY-775 (Cit + ACY-738 vs veh: $P<0.001$; Cit + ACY-775 vs veh: $P<0.01$; Figure $4 \mathrm{a})$. The potency of the effect of the combination with ACY-738 was comparable to that of a 40 -fold higher dose of citalopram administered alone.

We previously reported a pro-resilient phenotype in the mouse CSD paradigm after serotonin-selective KO of HDAC6 (Espallergues et al, 2012). Here we tested whether chronic pharmacological inhibition of HDAC6 replicates this phenotype. Mice were pre-treated for 10 days with either vehicle, fluoxetine $(20 \mathrm{mg} / \mathrm{kg}), \mathrm{ACY}-738(5 \mathrm{mg} / \mathrm{kg})$, or ACY-775 (50 mg/kg), and were exposed to 10 days of CSD, with continued treatment (Figure 4c). On day 21, $24 \mathrm{~h}$ after last injection, mice were tested in the social interaction test. Compared with undefeated controls, vehicle-treated mice exposed to CSD showed a significant decrease in time in the interaction zone in the presence of a social target (Figure $4 \mathrm{~d}$; $P<0.01)$ indicative of social avoidance. Treatment with fluoxetine, ACY-738 or ACY-775 prevented CSD-induced decrease in social interaction time. Defeated mice treated with ACY-738 showed significantly higher time in the interaction zone than those treated with vehicle $(P<0.05)$. Similar trends were seen with ACY-775 $(P=0.18)$ and fluoxetine $(P=0.08)$, albeit these effects did not reach posthoc significance. Similarly, treatment with ACY-738 led to a decrease in the amount of time spent in the corner zones (Figure 4e; $P<0.05 v s$ vehicle). Significant effects were also observed when data were evaluated in terms of proportion of mice reaching criterion for resiliency. In mice treated with vehicle, we observed $27 \%$ of mice to be spontaneously resilient; treatment with fluoxetine increased the percentage to $60 \%(P<0.05)$; ACY-738 to $81 \%(P<0.01)$; and ACY -775 to $58 \%(P=0.12$; Figure $4 \mathrm{f})$. Drug treatments did not change total distance traveled (Figure $4 \mathrm{~g}$ ) or interaction time in undefeated control mice (data not shown). There was no significant effect of ACY-738 or ACY-775 on body weight gain during the 4 weeks of treatment.

\section{DISCUSSION}

This study establishes the in vitro selectivity, pharmacokinetics, and psychoactive properties of two novel brainpenetrant small molecule inhibitors of HDAC6, namely ACY-738 and ACY-775.

Interest in HDAC6 as a possible therapeutic target in the CNS (d'Ydewalle et al, 2012) has grown considerably following discovery of the key role of this enzyme in aggresome formation (Kawaguchi et al, 2003), pathological evidence for its inclusion in Lewy bodies in Parkinson's disease as well as in glial cytoplasmic inclusions in frontotemporal lobar degeneration with TDP-43 inclusions (Ding et al, 2008), and evidence suggestive of a role in Alzheimer's disease (Govindarajan et al, 2012; Sung et al, 2013; Xiong et al, 2013).

Tubastatin A and its newer analogs, which have an excellent HDAC6 selectivity, are currently the reference HDAC6 inhibitors. These probes have shown therapeutic promise in disease models of Charcot Marie Tooth disease (d'Ydewalle et al, 2011) and Alzheimer's disease (Kim et al, 2012). To our knowledge, in vivo PK profiles of tubastatin A have not previously been reported. Our data suggest that after acute administration, at the dose tested here, tubastatin A has a limited brain biodistribution and does not lead to hyperacetylation of $\alpha$-tubulin in CNS, the most abundant and best-characterized substrate of HDAC6. This relative lack of CNS activity upon acute systemic administration was in contrast to the potent effects of tubastatin A observed in neuronal culture and peripheral tissues. This suggests that behavioral and neuroprotective effects of tubastatin A previously reported in in vivo models of neurodegenerative disease (Benner et al, 2012) could be independent of $\alpha$-tubulin acetylation or result from peripheral actions of the drug, such as the regulation of Foxp $3^{+}$regulatory T cells or other inflammatory mediators, which could indirectly affect neurodegenerative processes in brain (Vishwakarma et al, 2013; Beier et al, 2012).

For ACY-738 and ACY-775, we report an in vitro HDAC6 inhibitory potency in the low nanomolar range and an average selectivity of $100 \times$ over class I isoforms. Despite their rapid elimination from plasma, biodistribution, and pharmacodynamic data indicate that upon systemic administration, ACY-738 and ACY-775 lead to sufficient brain exposure to serve as useful pharmacological probes of HDAC6 function in the brain. Indeed, our results show that both compounds induce dramatic changes in the acetylated fraction of $\alpha$-tubulin persisting up to $4 \mathrm{~h}$ after acute systemic administration.

As predicted by our tissue culture results, the hyperacetylation of $\alpha$-tubulin observed in vivo after administration of behaviorally active dose ACY-738 was not accompanied by detectable increases in $\mathrm{H} 3 \mathrm{~K} 9$ acetylation globally, as measured by western blot, or by enrichment of H3K9 binding at promoter regions of activity-induced neuronal genes (BDNF and cFOS), as measured by ChIP. In contrast, administration of a behaviorally active dose of the class I 
HDAC inhibitor sodium butyrate, which has been reported to increase global H3 acetylation (Gundersen and Blendy, 2009), resulted in elevations of $\mathrm{H} 3$ acetylation at these promoters. These results are consistent with the large body of literature including our own previous observations in HDAC6 KOs supporting a limited contribution of HDAC6 to chromatin remodeling. As behavioral effects of ACY-738 and ACY-775 are observed as soon as $15 \mathrm{~min}$ after treatment, these effects are unlikely to reflect the consequence of alterations in gene transcription and de novo protein synthesis. Nevertheless, based on a small number of studies showing that in certain cell types such as T cells HDAC6 is present in the nucleus and associates with chromatin and promoters, we cannot formally exclude the possibility that ACY inhibitors may also exert some of their activity through epigenetic regulation of CNS genes that were not directly examined here. Genomewide analysis will be necessary to more definitively address this possibility.

It should be noted that despite its superior selectivity ACY-775 led to more variable results than ACY-738 in both the TST and social defeat paradigms. This is most likely attributable to 4.5-fold lower potency of ACY-775 and its more limited bioavailability possibly resulting in reduced target engagement. In addition, because of its poor solubility ACY-775 had to be given as a suspension at $50 \mathrm{mg} / \mathrm{kg}$, another factor likely increasing individual variability in drug exposure.

Along with others, we have shown previously that serotonin neurons in the DRN are enriched in HDAC6 and provided evidence that genetic ablation of HDAC6, either globally or conditionally in serotonin neurons, induces antidepressantlike phenotypes (Espallergues et al, 2012; Fukada et al, 2012). Our present results confirm pharmacologically that inhibition of HDAC6 is a plausible antidepressant strategy.

Our results show that ACY-738 and ACY-775 display rapid psychoactive properties upon acute and chronic administration. We observed acute exploration-enhancing effects of these compounds in novel environments as well as anxiolytic-like effects in the open-field, marble-burying, and NIH tests, but a lack of effect in the EPM, where acutely administered antidepressants are typically inactive. We also detected acute antidepressant-like anti-immobility effects in the TST, as well as chronic pro-resilience effects in mice exposed to CSD. Acute behavioral effects in the open field and TST are fully attributable to the selective inhibition of HDAC6 in CNS, as these responses are occluded in mice with a conditional neural KO of HDAC6. Our observation of antidepressant-like effects of ACY-738 and ACY-775 in CSD model following chronic treatment with $5 \mathrm{mg} / \mathrm{kg}$ (ie, a dose inactive in acute models) suggest the implication of distinct neurobiological mechanisms or a possible potentiation of the therapeutic effects with repeated administration.

The ambulation-enhancing effects of ACY drugs observed here in the open field are similar to previously reported hyperlocomotor effect of serotonergic antidepressants (Brocco et al, 2002). As these effects are not observed when mice were tested in a home cage environment, they likely reflect an increased motivation in a novel environment or reduction in neophobia as opposed to a nonspecific enhancement of motor function. Two additional lines of evidence support this conclusion: first, significant effects of ACY-738 and ACY-775 were observed in the marble- burying test, where reduction in active response (ie, burying) is used as primary readout for drug activity. Second, antidepressant-like responses in CSD were observed in mice tested $24 \mathrm{~h}$ after the last administration, a time-point when the drug has been fully eliminated from the organism.

Our observations of antidepressant-like activity of HDAC6 inhibitors is in line with the results of previous studies showing that compounds such as SAHA, that have nм affinity for HDAC6, also produce antidepressant-like responses in mice (Covington et al, 2009). The observation that MS-275, a class I-selective inhibitor, produces antidepressant-like effects when infused in the brain suggests that both histone-dependent and -independent mechanisms likely contribute to the activity of these drugs (Covington et al, 2011; Golden et al, 2013).

Whether acetylation of $\alpha$-tubulin, which is regulated by HDAC6 but not by class I HDACs, has a causal role in the activity of HDAC6 inhibitors remains an open question. It should be noted, however, that the class I selectivity of MS-275 does not necessarily imply an epigenetic selectivity in its mode of action, as large scale acetylation-proteomics studies have shown that MS-275 induces the hyperacetylation of numerous non-histone proteins (Choudhary et al, 2009), some of which are also hyperacetylated after ACY738 administration or KO of HDAC6. The chaperone protein Hsp90, which has an important role in glucocorticoid receptor signaling, is one such common non-histone target of class I and class IIb HDACs that could plausibly mediate the common effects of class I and II HDAC inhibitors in stress resilience (Beier et al, 2012; de Zoeten et al, 2011; Espallergues et al, 2012; Nishioka et al, 2008).

Two major limitations of current antidepressants in clinical settings are the variability of response and delayed mode of action. This variability and delayed action are also observed in certain preclinical models like the CSD. Our results suggest that, when given prophylactically before and during exposure to CSD, HDAC6-selective inhibitors produce stress protective effects that appear more robust than those of the traditional SSRI fluoxetine. Furthermore, our observation that combination of a behaviorally inactive dose of ACY-738 with a subactive dose of citalopram produces behavioral effects equivalent to that of a 40 -fold higher dose of citalopram administered alone, suggests a convergence of the downstream intracellular mechanisms engaged by SSRIs and HDAC6 inhibition. Our observations that a pan-neuronal HDAC6 KO potentiates SSRI activity suggests that HDAC6 blockade may act in part by lifting an inhibitory influence on serotonin-mediated signaling. This conclusion is further supported by a previous report showing that fluoxetine and global HDAC6 KO have synergistic antidepressant-like effects in mice (Fukada et al, 2012). Unexpectedly, we found that mice with Nestin-driven HDAC6 KO lack the baseline antidepressant and hyperlocomotor phenotype reported earlier in the global HDAC6 KO mice. These strain differences are likely to reflect cell-type and/or region-specific effects of HDAC6 and compensatory mechanisms dependent on the developmental period of the KO.

Taken together with the positive side-effect profiles of HDAC6 inhibitors (Raje et al, 2013), these results suggest that pharmacological HDAC6 inhibition may provide a 
useful approach to reduce rates of non-response and potentiate activity of currently available antidepressant treatments such as SSRIs.

Further studies will be necessary to better define the time courses and potential persistence of the antidepressant-like activity of HDAC6 inhibitors as well as their downstream mechanism of action. A likely mechanism may involve the Hsp90 chaperone complex and regulation of microtubuledependent trafficking in axons and dendrites. Pharmacologic or genetic inhibition of HDAC6 has been shown to increase the trafficking of mitochondria (Chen et al, 2010) and dense core vesicles containing BDNF (Dompierre et al, 2007 ) in neurons. Along with others, we have shown that inhibition or genetic ablation of HDAC6 modulates nuclear trafficking of GR in response to glucocorticoid hormones and reduces stress signaling in DRN serotonin and prefrontal cortex pyramidal neurons, thereby promoting resilience (Espallergues et al, 2012; Lee et al, 2012). Tools developed and validated in this study will offer fundamentally novel opportunities to dissect the role of HDAC6 in cognitive and affective aspects of stress resilience.

\section{FUNDING AND DISCLOSURE}

OB is inventor on patent application \#61713014 'Pyrimidine hydroxyamide compounds as protein deacetylase inhibitors and methods of use thereof '. OB has received financial research support from Acetylon Pharmaceuticals. JHVD and MJ are employees of Acetylon Pharmaceuticals. RM is cofounder and member of the scientific advisory board of Acetylon Pharmaceuticals. RM has financial interests in SHAPE Pharmaceuticals and Acetylon Pharmaceuticals. He is also the inventor on IP licensed to these two entities. RM's interests were reviewed and are managed by Massachusetts General Hospital and Partners HealthCare in accordance with their conflict of interest policies. JHVD and MJ and SJ are shareholders in Acetylon Pharmaceuticals. The remaining authors declare no conflict of interest.

\section{ACKNOWLEDGEMENTS}

We thank A Kozikowski for providing tubastatin A and I Lucki for his guidance and valuable input on the manuscript. This work was supported by grants MH087581 from the US National Institute of Mental Health and an award from the International Mental Health Research Organization (IMHRO) to $\mathrm{OB}$ and by training grant GM008076-29 from NIH.

\section{Author contributions}

JJ designed and performed the experiments, collected, analyzed and interpreted the data, and wrote the manuscript. JB performed the experiments. BGL designed and performed the experiments and interpreted data. $\mathrm{JAB}$ provided environment and support. MJ, JHVD, and RM contributed vital, novel reagents, and interpreted data. $\mathrm{OB}$ designed research, interpreted data, provided environment, and support and wrote the manuscript.

\section{REFERENCES}

Albelda N, Joel D (2012). Animal models of obsessive-compulsive disorder: exploring pharmacology and neural substrates. Neurosci Biobehav Rev 36: 47-63.

Beier UH, Wang L, Han R, Akimova T, Liu Y, Hancock WW (2012). Histone deacetylases 6 and 9 and sirtuin-1 control Foxp3 + regulatory T cell function through shared and isoformspecific mechanisms. Sci Signaling 5: ra45-ra45.

Benner L, Kalin JH, Kozikowski AP, Gordon MN, Morgan D, SM-L B (2012). Selective HDAC6 inhibition decreases tau levels and improves spatial memory deficits in the $\mathrm{rtg} 4510$ mouse model. Society Neurosc 2012, Program No. 150.04 http://www. abstractsonline.com/Plan/ViewAbstract.aspx?sKey=804c6b94-1e7749c4-80c5-4098b4c64f01\&cKey $=440038 \mathrm{~d} 9-3320-4 \mathrm{f} 02-916 \mathrm{~d}-7 \mathrm{e} 5 \mathrm{c} 2$ 4968839\&mKey $=\{70007181-01 C 9-4 D E 9-A 0 A 2-E E B F A 14 C D 9 F 1\}$.

Berton O, McClung CA, Dileone RJ, Krishnan V, Renthal W, Russo SJ et al (2006). Essential role of BDNF in the mesolimbic dopamine pathway in social defeat stress. Science 311: 864-868.

Bertrand P (2010). Inside HDAC with HDAC inhibitors. Eur J Med Chem 45: 2095-2116.

Bradner JE, West N, Grachan ML, Greenberg EF, Haggarty SJ, Warnow $\mathrm{T}$ et al (2010). Chemical phylogenetics of histone deacetylases. Nat Chem Biol 6: 238-243.

Brocco M, Dekeyne A, Veiga S, Girardon S, Millan MJ (2002). Induction of hyperlocomotion in mice exposed to a novel environment by inhibition of serotonin reuptake. A pharmacological characterization of diverse classes of antidepressant agents. Pharmacol Biochem Behav 71: 667-680.

Broekkamp CL, Rijk HW, Joly-Gelouin D, Lloyd KL (1986). Major tranquillizers can be distinguished from minor tranquillizers on the basis of effects on marble burying and swim-induced grooming in mice. Eur J Pharmacol 126: 223-229.

Butler KV, Kalin J, Brochier C, Vistoli G, Langley B, Kozikowski AP (2010). Rational design and simple chemistry yield a superior, neuroprotective HDAC6 inhibitor, tubastatin A. J Am Chem Soc 132: 10842-10846.

Chen S, Owens GC, Makarenkova H, Edelman DB (2010). HDAC6 regulates mitochondrial transport in hippocampal neurons. PLoS One 5: e10848.

Choudhary C, Kumar C, Gnad F, Nielsen ML, Rehman M, Walther TC et al (2009). Lysine acetylation targets protein complexes and co-regulates major cellular functions. Science 325: 834-840.

Covington HE, Maze I, Laplant QC, Vialou VF, Ohnishi YN, Berton O et al (2009). Antidepressant actions of histone deacetylase inhibitors. J Neurosci 29: 11451-11460.

Covington HE, Vialou VF, Laplant Q, Ohnishi YN, Nestler EJ (2011). Hippocampal-dependent antidepressant-like activity of histone deacetylase inhibition. Neurosci Lett 493: 122-126.

d'Ydewalle C, Bogaert E, Van Den Bosch L (2012). HDAC6 at the intersection of neuroprotection and neurodegeneration. Traffic 13: 771-779.

d'Ydewalle C, Krishnan J, Chiheb DM, Van Damme P, Irobi J, Kozikowski AP et al (2011). HDAC6 inhibitors reverse axonal loss in a mouse model of mutant HSPB1-induced CharcotMarie-Tooth disease. Nat Med 17: 968-974.

de Zoeten EF, Wang L, Butler K, Beier UH, Akimova T, Sai H et al (2011). Histone deacetylase 6 and heat shock protein 90 control the functions of Foxp3 $(+)$ T-regulatory cells. Mol Cell Biol 31: 2066-2078.

Ding H, Dolan PJ, Johnson GVW (2008). Histone deacetylase 6 interacts with the microtubule-associated protein tau. J Neurochem 106: 2119-2130.

Dompierre JP, Godin JD, Charrin BC, Cordelieres FP, King SJ, Humbert $S$ et al (2007). Histone deacetylase 6 inhibition compensates for the transport deficit in Huntington's disease by increasing tubulin acetylation. J Neurosci 27: 3571-3583. 
Espallergues J, Teegarden SL, Veerakumar A, Boulden J, Challis C, Jochems J et al (2012). HDAC6 regulates glucocorticoid receptor signaling in serotonin pathways with critical impact on stress resilience. J Neurosci 32: 4400-4416.

Fukada M, Hanai A, Nakayama A, Suzuki T, Miyata N, Rodriguiz RM et al (2012). Loss of deacetylation activity of HDAC6 affects emotional behavior in Mice. PLoS One 7: e30924.

Golden SA, Christoffel DJ, Heshmati M, Hodes GE, Magida J, Davis K et al (2013). Epigenetic regulation of RAC1 induces synaptic remodeling in stress disorders and depression. Nat Med 19: 337-344.

Golden SA, Covington HE, Berton O, Russo SJ (2011). A standardized protocol for repeated social defeat stress in mice. Nat Protocols 6: 1183-1191.

Govindarajan N, Rao P, Burkhardt S, Sananbenesi F, Schlüter OM, Bradke F et al (2012). Reducing HDAC6 ameliorates cognitive deficits in a mouse model for Alzheimer's disease. EMBO Mol Med 5: 52-63.

Guan J-S, Haggarty SJ, Giacometti E, Dannenberg J-H, Joseph N, Gao J et al (2009). HDAC2 negatively regulates memory formation and synaptic plasticity. Nature 459: 55-60.

Gundersen BB, Blendy JA (2009). Neuropharmacology. Neuropharmacology 57: 67-74.

Haberland M, Montgomery RL, Olson EN (2009). The many roles of histone deacetylases in development and physiology: implications for disease and therapy. Nat Rev Genet 10: 32-42.

Haggarty SJ, Koeller KM, Wong JC, Grozinger CM, Schreiber SL (2003). Domain-selective small-molecule inhibitor of histone deacetylase 6 (HDAC6)-mediated tubulin deacetylation. Proc Natl Acad Sci USA 100: 4389-4394.

Hobara T, Uchida S, Otsuki K, Matsubara T, Funato H, Matsuo K et al (2010). Altered gene expression of histone deacetylases in mood disorder patients. J Psychiatric Res 44: 263-270.

Hubbert C, Guardiola A, Shao R, Kawaguchi Y, Ito A, Nixon A et al (2002). HDAC6 is a microtubule-associated deacetylase. Nature 417: 455-458.

Kawaguchi Y, Kovacs JJ, McLaurin A, Vance JM, Ito A, Yao TP (2003). The deacetylase HDAC6 regulates aggresome formation and cell viability in response to misfolded protein stress. Cell 115: 727-738.

Kennedy PJ, Feng J, Robison AJ, Maze I, Badimon A, Mouzon E et al (2013). Class I HDAC inhibition blocks cocaine-induced plasticity by targeted changes in histone methylation. Nat Neurosci 16: 434-440.

Kim C, Choi H, Jung ES, Lee W, Oh S, Jeon NL et al (2012). HDAC6 inhibitor blocks amyloid beta-induced impairment of mitochondrial transport in hippocampal neurons. PLoS One 7: e42983.

Kovacs JJ, Murphy PJM, Gaillard S, Zhao X, Wu J-T, Nicchitta CV et al (2005). HDAC6 regulates Hsp90 acetylation and chaperonedependent activation of glucocorticoid receptor. Mol Cell 18: 601-607.

Lee JB, Wei J, Liu W, Cheng J, Feng J, Yan Z (2012). Histone deacetylase 6 gates the synaptic action of acute stress in prefrontal cortex. J Physiol 590: 1535-1546.

Li Y, Zhang X, Polakiewicz RD, Yao T-P, Comb MJ (2008). HDAC6 is required for epidermal growth factor-induced beta-catenin nuclear localization. J Biol Chem 283: 12686-12690.

Lister RG (1987). The use of a plus-maze to measure anxiety in the mouse. Psychopharmacologia 92: 180-185.

Lucki I, Dalvi A, Mayorga A (2001). Sensitivity to the effects of pharmacologically selective antidepressants in different strains of mice. Psychopharmacology 155: 315-322.

Mao X, Hou T, Cao B, Wang W, Li Z, Chen S et al (2011). The tricyclic anti-depressant amitriptyline inhibits D-cyclin transactivation and induces myeloma cell apoptosis by inhibiting histone deacetylases: in vitro and in silico evidence. Mol Pharmacol 79: 672-680.
Merali Z, Levac C, Anisman H (2003). Validation of a simple, ethologically relevant paradigm for assessing anxiety in mice. BPS 54: 552-565.

Nasca C, Xenos D, Barone Y, Caruso A, Scaccianoce S, Matrisciano F et al (2013). L-acetylcarnitine causes rapid antidepressant effects through the epigenetic induction of mGlu2 receptors. Proc Natl Acad Sci USA 110: 4804-4809.

Nishioka CC, Ikezoe TT, Yang JJ, Takeuchi SS, Koeffler HPH, Yokoyama AA (2008). MS-275, a novel histone deacetylase inhibitor with selectivity against $\mathrm{HDAC1}$, induces degradation of FLT3 via inhibition of chaperone function of heat shock protein 90 in AML cells. Leuk Res 32: 11.

Phiel CJ, Zhang F, Huang EY, Guenther MG, Lazar MA, Klein PS (2001). Histone deacetylase is a direct target of valproic acid, a potent anticonvulsant, mood stabilizer, and teratogen. J Biol Chem 276: 36734-36741.

Raje N, Hari PN, Vogl DT, Jagannath S, Orlowski RZ, Supko JG et al (2013). Rocilinostat (ACY-1215), a selective HDAC6 inhibitor, alone and in combination with bortezomib in multiple myeloma: preliminary results from the first-in-humans phase I/II study. Abstracts of the 54th Annual meeting of the American Society of Hematology. Atlanta, Georgia, 2012. Available online here https://ash.confex.com/ash/2012/webprogram/Paper52013.html.

Rivieccio MA, Brochier C, Willis DE, Walker BA, D'Annibale MA, McLaughlin $\mathrm{K}$ et al (2009). HDAC6 is a target for protection and regeneration following injury in the nervous system. Proc Natl Acad Sci USA 106: 19599-19604.

Russo SJ, Charney DS (2013). Next generation antidepressants. Proc Natl Acad Sci USA 110: 4441-4442.

Santo L, Hideshima T, Kung AL, Tseng J-C, Tamang D, Yang M et al (2012). Preclinical activity, pharmacodynamic, and pharmacokinetic properties of a selective HDAC6 inhibitor, ACY-1215, in combination with bortezomib in multiple myeloma. Blood 119: 2579-2589.

Sung YM, Lee T, Yoon H, DiBattista AM, Song JM, Sohn Y et al (2013). Mercaptoacetamide-based class II HDAC inhibitor lowers $A \beta$ levels and improves learning and memory in a mouse model of Alzheimer's disease. Exp Neurol 239: 192-201.

Verdel A, Curtet S, Brocard MP, Rousseaux S, Lemercier C, Yoshida M et al (2000). Active maintenance of mHDA2/mHDAC6 histonedeacetylase in the cytoplasm. Curr Biol 10: 747-749.

Vishwakarma S, Iyer LR, Muley M, Singh PK, Shastry A, Saxena A et al (2013). Tubastatin, a selective histone deacetylase 6 inhibitor shows anti-inflammatory and anti-rheumatic effects. Int Immunopharmacol 16: 1-7.

White LA, Eaton MJ, Castro MC, Klose KJ, Globus MY, Shaw G et al (1994). Distinct regulatory pathways control neurofilament expression and neurotransmitter synthesis in immortalized serotonergic neurons. J Neurosci 14: 6744-6753.

Xiong Y, Zhao K, Wu J, Xu Z, Jin S, Zhang YQ (2013). HDAC6 mutations rescue human tau-induced microtubule defects in Drosophila. Proc Natl Acad Sci USA 110: 4604-4609.

Zhang L, Rubins NE, Ahima RS, Greenbaum LE, Kaestner KH (2005). Foxa2 integrates the transcriptional response of the hepatocyte to fasting. Cell Metab 2: 141-148.

Zhang X, Yuan Z, Zhang Y, Yong S, Salas-Burgos A, Koomen J et al (2007). HDAC6 modulates cell motility by altering the acetylation level of cortactin. Mol Cell 27: 197-213.

Zhang Y, Kwon S, Yamaguchi T, Cubizolles F, Rousseaux S, Kneissel $\mathrm{M}$ et al (2008). Mice lacking histone deacetylase 6 have hyperacetylated tubulin but are viable and develop normally. Mol Cell Biol 28: 1688-1701.

Zhang Y, Li N, Caron C, Matthias G, Hess D, Khochbin S et al (2003). HDAC-6 interacts with and deacetylates tubulin and microtubules in vivo. EMBO J 22: 1168-1179.

Supplementary Information accompanies the paper on the Neuropsychopharmacology website (http://www.nature.com/npp) 